\title{
Clinicopathological Study of Resections of Intraductal Papillary Neoplasm of the Bile Duct
}

\author{
TOSHIHIRO SATO ${ }^{1}$, TORU HISAKA ${ }^{1}$, HISAMUNE SAKAI ${ }^{1}$, HIROTO ISHIKAWA ${ }^{1}$, \\ RYUICHI KAWAHARA ${ }^{1}$, YUICHI GOTO ${ }^{1}$, YORIKO NOMURA ${ }^{1}$, MASAFUMI YASUNAGA ${ }^{1}$, \\ FUMIHIKO FUJITA $^{1}$, MASAHIKO TANIGAWA ${ }^{2}$, YOSHIKI NAITO ${ }^{2}$, JUN AKIBA ${ }^{2}$, \\ HIROHISA YANO $^{3}$, HIROYUKI TANAKA ${ }^{1}$, YOSHITO AKAGI ${ }^{1}$ and KOJI OKUDA ${ }^{1}$ \\ Departments of ${ }^{1}$ Surgery and ${ }^{3}$ Pathology, Faculty of Medicine, Kurume University, Kurume, Japan \\ ${ }^{2}$ Department of Diagnostic Pathology, Kurume University Hospital, Kurume, Japan
}

\begin{abstract}
Background/Aim: The aim of this study was to examine the clinicopathological features of intraductal papillary neoplasm of the bile duct (IPNB) and investigate their relationships with intraductal papillary mucinous neoplasm (IPMN). Patients and Methods: Our study included 104 patients who underwent resection of tumors that showed papillary growth within the bile duct and pancreas. Results: Comparisons were performed based on subtypes and histological grades. The presence of various histological grades was confirmed in both the IPNB group and the IPMN group, and statistical significance was found in the betweengroup comparisons of subtypes and histological grades. It was shown that while all patients who underwent IPNB resection did not match the classifications proposed by Nakanuma et al., they did reflect classification characteristics. Conclusion: $I P N B$ and IPMN have common clinical histological features. Common features between IPNB subtype classifications were also identified, which may provide novel diagnostics.
\end{abstract}

Intraductal papillary neoplasm of the bile duct (IPNB) is an epithelial tumor that shows papilloma within a dilated bile duct and was first reported by Chen et al. in 2001 (1). Histologically, IPNB are well-differentiated papillary adenocarcinomas or borderline lesions and have been reported to resemble intraductal papillary mucinous neoplasm (IPMN) (2). IPMN is

This article is freely accessible online.

Correspondence to: Toru Hisaka, MD, Ph.D., Department of Surgery, Kurume University Faculty of Medicine, 67 Asahi-machi, Kurume, Fukuoka 830-0011, Japan. Tel: +81 942317566, Fax: +81 942340709, e-mail: thisaka@med.kurume-u.ac.jp

Key Words: Intraductal papillary neoplasm of the bile duct (IPNB), intraductal papillary mucinous neoplasm (IPMN), subtypes, clinicopathological study. an epithelial tumor that displays papilloma accompanied by mucin hypersecretion. IPMN is sub-classified according to macroscopic characteristics, tissue morphology, and mucoidtrait expression. IMPN is reported to have different malignancy levels, prognoses, recurrence rates, etc. (3). While there is consensus regarding disease concept, diagnosis, and treatment of IPMN, sufficient consensus does not exist for IPNB because it is a relatively new disease concept. Conventionally, bile duct and pancreatic diseases have been classified from separate viewpoints. Although the bile duct and pancreas have anatomical and embryological similarities, IPNB has been considered to be a counterpart of IPMN, and this logic is convincing. Nevertheless, IPNB does not occur as frequently as IPMN, and many uncertainties remain. The purpose of the present study was to clarify the clinicopathological features of IPNB and elucidate their relationship with pancreatic IPMN.

\section{Patients and Methods}

During our study period, from 2003 through 2015, 104 patients at the Kurume University Hospital received resections for papillary growth tumors within the bile duct and pancreas. After fixing the excised specimens in $10 \%$ neutral buffered formalin solution, sections of $5 \mathrm{~mm}$ thickness were prepared, and hematoxylin-eosin (HE) staining was performed. The stained sections were clinicopathologically investigated using microscopy. The clinicopathological investigation was performed in reference to the WHO Classification of Tumors of the Digestive System, 4th edition (4-6). The IPNB sub-classifications were performed according to the classifications reported by Fukumura et al. (7).

Immunohistochemistry. Immunocytochemistry was performed on 4$\mu \mathrm{m}$-thick sections of formalin-fixed, paraffin embedded tissues. The sections were mounted on glass slides and then incubated with antimouse monoclonal antibody against human MUC-1 (clone Ma695, IgG1, 1:100 dilution; Leica Biosystems Newcastle, Ltd., Newcastle, UK), anti-mouse monoclonal antibody against human MUC-2 (clone Cep58, IgG1, 1:100 dilution; Leica Biosystems), anti-mouse monoclonal antibody against human MUC-5AC (clone CLH2, IgG1 1:100 dilution; Leica Biosystems), or anti-mouse monoclonal 
antibody against human MUC-6 (clone CLH5, IgG1 1:100 dilution; Leica Biosystems) on the same fully automated Bond-III system (Leica Microsystems) using onboard heat-induced antigen retrieval with epitope retrieval solution 2 for $20 \mathrm{~min}$ at $99^{\circ} \mathrm{C}$. Each slide was incubated with the antibody for $30 \mathrm{~min}$ at room temperature. This automated system uses a Refine polymer detection kit with horseradish peroxidase-polymer as a secondary antibody and DAB, and incubation with a secondary antibody was performed for $30 \mathrm{~min}$ at room temperature (Figure 1).

All Immunohistochemical analyses were evaluated by two experienced observers who were unaware of the patients' conditions.

Statistical analysis. Clinicopathologic factors were compared between the two groups, the IPNB and IPMN group, using the Student's $t$-test, chi-squared test, and Kruskal-Wallis test. Overall survival (OS) curves were created for both groups using the Kaplan-Meier method, and the log-rank test was used to examine the survival curves. The significance level was set at $p<0.05$, and JMP Pro 11.0.0 (SAS Institute Inc., Cary, NC, USA) was used for the analysis.

\section{Results}

There were 28 cases in the IPNB group including intracholecystic papillary neoplasm (ICPN) in the gallbladder and 76 cases in the IPMN group. Of these patients, 24 with IPNB and 74 with IPMN were suitable for histopathological examination. The patients with IPNB ranged from 49 to 83 years (mean 68.2 years) in age, with a male:female ratio of 20:8. The patients with IPMN ranged in age from 51 to 82 years (mean 66.7 years), with a male:female ratio of 57:19. Of the IPNB cases, 5 were intrahepatic and 23 were extrahepatic (gallbladder 5 cases). The following IPNB surgical treatments were performed: 13 cases received pancreatoduodenectomy (PD), 5 cases hepatectomy, 4 cases hepatectomy with biliary resection, and 5 cases cholecystectomy with liver bed resection. The following surgical treatments were performed for IPMN: 34 cases received PD, 25 cases distal pancreatectomy (DP), and 9 cases total pancreatectomy (TP).

With respect to mucin hypersecretion, patients with IPMN (79\%) exhibited predominantly higher levels of mucin than patients with IPNB (36\%). The IPNB sub-classification breakdown was as follows: 7 cases of gastric type, 3 cases of intestinal type, 13 cases of pancreatobiliary type, and 1 case of oncocytic type. The IPMN sub-classification breakdown was as follows: 26 cases of gastric type, 35 cases of intestinal type, 10 cases of pancreatobiliary type, and 3 cases of oncocytic type. No significant differences were found in the sub-classification comparisons between the two groups. The nodule size was $3.34 \pm 0.41 \mathrm{~cm}$ in patients with IPNB and $2.50 \pm 0.23 \mathrm{~cm}$ in patients with IPMN, and this difference was not statistically significant.

The breakdown of histological grade was as follows. IPNB: no patients had low to intermediate-grade dysplasia, 13 patients had a high-grade dysplasia, and 11 patients had
IPNB-derived invasive carcinoma; IPMN: 22 patients had low to intermediate-grade dysplasia, 16 patients had a highgrade dysplasia, and 36 patients had IPMN-derived invasive carcinoma. Significant differences were found in the comparisons of each histological grade between groups. Within the nodules of patients in the IPMN group, different histological atypias were confirmed, while no such findings were observed in the IPNB group (data not shown). With respect to lymphatic invasion, neural invasion, and lymph node metastasis, while significant differences were not confirmed between the groups, the IPNB group displayed significantly higher venous invasion (Table I). Regarding prognosis, while the IPNB group had 5-year overall survival rate of $100 \%$, the 5-year survival rate for the IPMN group was $75 \%$. However, this difference was not statistically significant (Figure 2).

Nakanuma et al. proposed two sub-classifications based on histopathological findings (8), and we investigated 28 cases of IPNB resection based on these sub-classifications. While there were 5 cases of Type 1 IPNB, the majority of which occurred in the intrahepatic bile duct, 4 of the 5 cases had mucin hypersecretion, with the following subtypes: 2 case of pancreatobiliary type, 2 cases of gastric type, and 1 case of oncocytic type. Invasive cancer was found in 1 of the 5 cases. There were 23 cases of type 2 IPNB, all of which were of extrahepatic origin. Mucin hypersecretion was confirmed in 3 cases, and the cases were sub-classified as follows: 13 cases of pancreatobiliary type, 4 cases of gastric type, and 3 cases of intestinal type. Invasive cancer was found in 10 of the 23 cases.

\section{Discussion}

Regarding age and sex comparisons, no significant differences were found between the IPMN and IPNB groups. Mucin hypersecretion was more frequently noted in the IPMN group, and these findings were similar to those reported by another study (7). There were, however, major differences in mucus-trait type proportions between the two groups, with the IPNB showing nearly half the amount of MUC-1 expression relative to IPMN. Conversely, in the IPMN group, frequent MUC-2 or MUC-5AC expression was observed, thus demonstrating the differences in mucusexpression types. These differences may be reflected in sub-classification by types: while the IPNB group had many pancreatobiliary types, the IPMN group had many intestinal and gastric types. Gastric type IPMN had the second-most sub-classification numbers. Since the treatment plans have been established for gastric type IPMN (9), which comprises the majority of branch duct IPMN, the rate of resection may be reduced in the future. If this indeed proves true, then changes in intergroup proportions may present as well. While no significant 
A

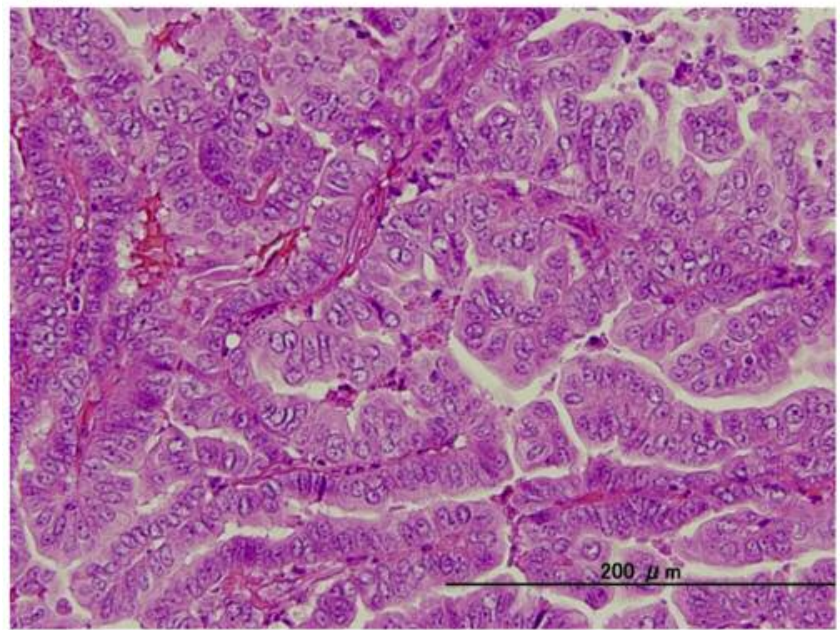

C

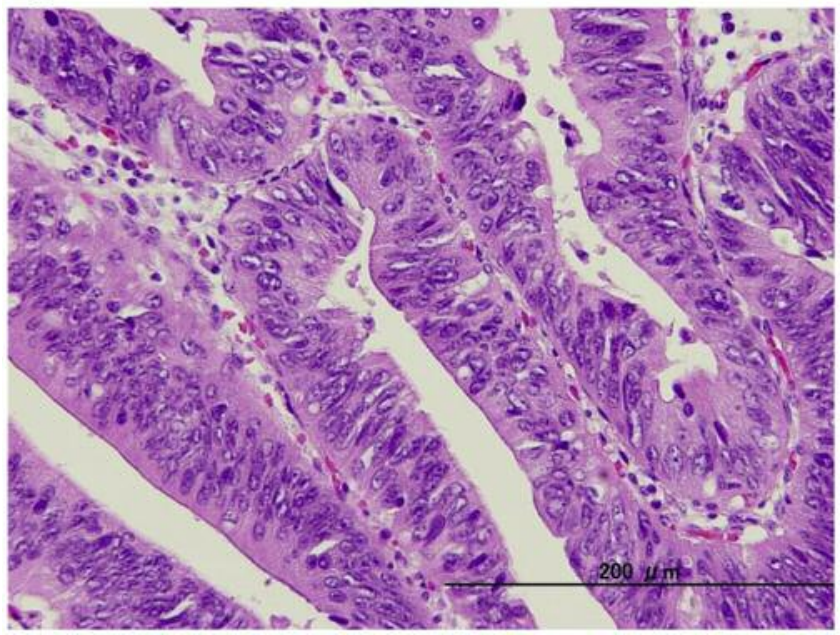

B

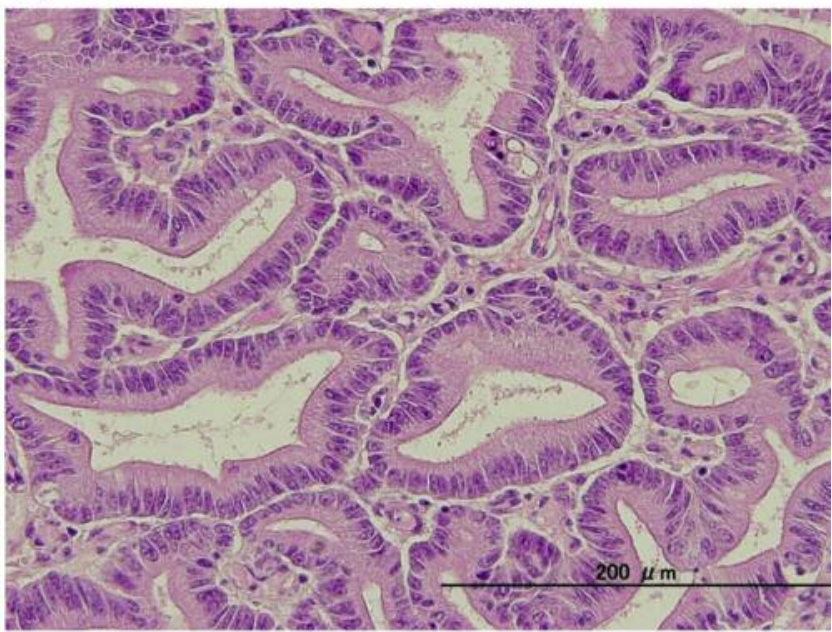

D

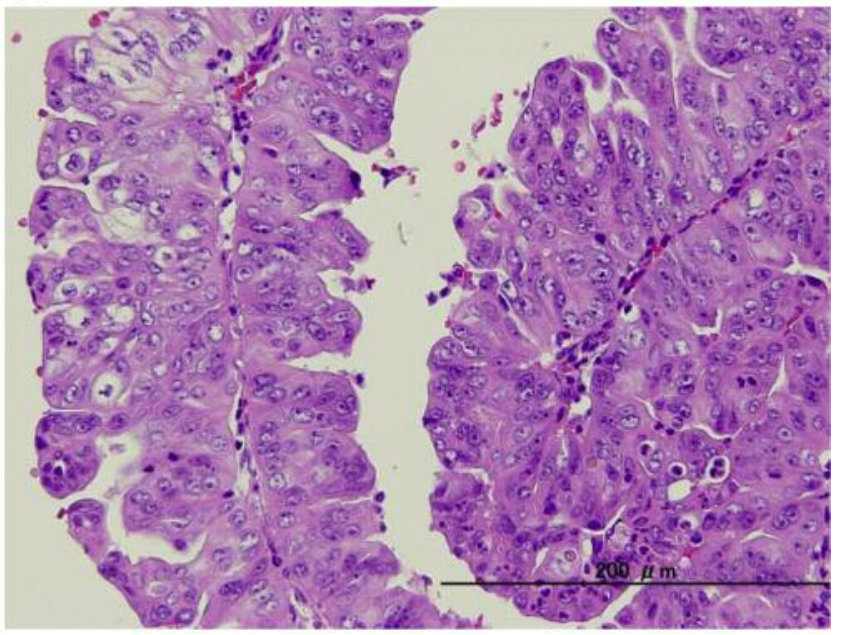

Figure 1. Morphological types of IPMN (×200). (A) Pancreatobiliary type, (B) gastric type, (C) intestinal type, (D) oncocytic type.

differences were found between the two groups in nodule size, a significant difference was found in the proportions of histological atypias.

A variety of histological atypias were found in the IPMN group. For IPNB, while high-grade dysplasia to invasive IPNB types were found, no low to intermediate-grade dysplasia was observed. There are two possible reasons for this. First, while the IPMN group had relatively larger numbers of adenoma, given this group's larger numbers of gastric and intestinal types, the IPNB group had conversely more pancreatobiliary types (3), with relatively higher malignancies. Secondly, a relatively higher number of patients with type 2 IPNB (so-called papillary carcinoma or cholangiocarcinoma) could have been included in the IPNB group (8). To minimize this possibility, we based our investigation on the classifications proposed by Nakanuma et al. (8) for the 28 cases of IPNB resection. Type 1 IPNB was found to be frequently present as intrahepatic, and mucin hypersecretion was also often observed. There were few invasive cases, and their morphology was extremely similar to that of IPMN. Meanwhile, type 2 IPNB was often found in the extrahepatic bile duct, rarely presenting mucin hypersecretion, and often exhibiting invasion. Thus, the morphology of type 2 IPNB does not resemble that of IPMN. While not all of the resection cases that were investigated in this study matched these profiles, their features were in fact reflected (Table II).

In our present study, both groups had favorable 5-year survival rates. This finding is similar to that of previous studies, especially in the survival rates of patients in the IPNB group 
Table I. Background of patients and tumors, and evaluation of tumors.

\begin{tabular}{|c|c|c|c|}
\hline & $\begin{array}{l}\text { IPMN } \\
(\mathrm{n}=76)\end{array}$ & $\begin{array}{l}\text { IPNB (ICPN) } \\
\quad(\mathrm{n}=28)\end{array}$ & $p$-Value \\
\hline Age (range) & $66.7(51-82)$ & $68.2(49-83)$ & \\
\hline Gender $(\mathrm{M} / \mathrm{F})$ & $57 / 19$ & $20 / 8$ & \\
\hline Location & $\begin{array}{l}\text { Branch } 27 \\
\text { Main } 40 \\
\text { Mix } 9\end{array}$ & $\begin{array}{c}\text { Intrahepatic } 5 \\
\text { Extrahepatic } 23 \text { (ICPN 5) }\end{array}$ & \\
\hline Surgical treatment & $\begin{array}{l}\text { PD } 34 \\
\text { DP } 25 \\
\text { TP } 9\end{array}$ & $\begin{array}{c}\text { PD } 13 \\
\text { HT } 5 \\
\text { HT (with biliary resection) } 4 \\
\text { CT } 5\end{array}$ & \\
\hline Mucin hypersecretion & $60(79 \%)$ & $10(36 \%)$ & $<0.0001$ \\
\hline Subtype & & & 0.0004 \\
\hline Gastric & 26 & 7 & \\
\hline Intestinal & 35 & 3 & \\
\hline Pancreatobiliary & 10 & 13 & \\
\hline Oncocytic & 3 & 1 & \\
\hline Tumor size $(\mathrm{cm})$ & $2.50 \pm 0.23$ & $3.34 \pm 0.41$ & 0.085 \\
\hline Histological grade & & & $<0.0001$ \\
\hline LID & 22 & 0 & \\
\hline HGD & 16 & 13 & \\
\hline Invasive carcinoma & 36 & 11 & \\
\hline $\operatorname{Ly}(-/+)$ & $39 / 9$ & $15 / 6$ & 0.47 \\
\hline $\mathrm{V}(-/+)$ & $43 / 4$ & $15 / 7$ & 0.028 \\
\hline $\mathrm{Ne}(-/+)$ & $39 / 8$ & $19 / 3$ & 0.65 \\
\hline Nodal Stage pn $(0 / 1)$ & $41 / 6$ & $8 / 3$ & 0.26 \\
\hline
\end{tabular}

PD: Pancreatoduodenectomy; DP: distal pancreatectomy; TP: total pancreatectomy; HT: hepatectomy; CT: cholecystectomy with liver bed resection; LID: low-to intermediate-grade dysplasia; HGD: high-grade dysplasia; Ly: lymphatic invasion; V: venous invasion; Ne: neural invasion.

Table II. Pathological features of two types of biliary papillary neoplasms.

Type1 IPNB (n=5) Type2 IPNB (n=23)

\begin{tabular}{lcc}
\hline Location & Intrahepatic 5 & Extrahepatic 23 \\
Mucin hypersecretion & $4 / 5$ & $3 / 23$ \\
Subtype & Pancreatobiliary 2 & Pancreatobiliary 13 \\
& Gastric 2 & Gastric 4 \\
& Intestinal 0 & Intestinal 3 \\
Oncocytic 1 & Oncocytic 0 \\
Associated with invasive cancer & $1 / 5$ & $10 / 23$ \\
\hline
\end{tabular}

IPNB: Intraductal papillary neoplasms of the bile duct.

(10). Since a clear consensus does not exist for IPNB diagnosis and treatment, as is the case for IPMN, these issues must be clarified in the future. Because of the poor prognosis of invasive cancers $(11,12)$, IPNB should be resected in a stage when it still remains only intraepithelial. While it is currently difficult to obtain definitive presurgical findings, reports have indicated the possible usefulness of ${ }^{18} \mathrm{~F}$-fluolodeoxyglucose $\left({ }^{18} \mathrm{~F}-\mathrm{FDG}\right)$ examination $(13,14)$. In presurgical FDG-PET/CT exams, as malignancy rates increase, $\mathrm{SUV}_{\max }$ has been found to increase in ${ }^{18} \mathrm{~F}$-FDG tests, which indicates the possibility that this method to be useful for determining malignancy. Further investigations will establish clear-cut IPNB diagnosis and treatment strategies.

In conclusion, IPNB presented clinical histological features that are common to those of IPMN. The common features of IPNB subtype classifications (8) may provide novel diagnostic criteria.

\section{Conflicts of Interest}

The Authors declare no conflicts of interest associated with this manuscript.

\section{Authors' Contributions}

Toshihiro Sato designed the study, and wrote the initial draft of the manuscript. Toshihiro Sato and Toru Hisaka contributed to the analysis and interpretation of data, and assisted in the preparation of the manuscript. All other Authors have contributed to data collection and interpretation, and critically reviewed the manuscript. 


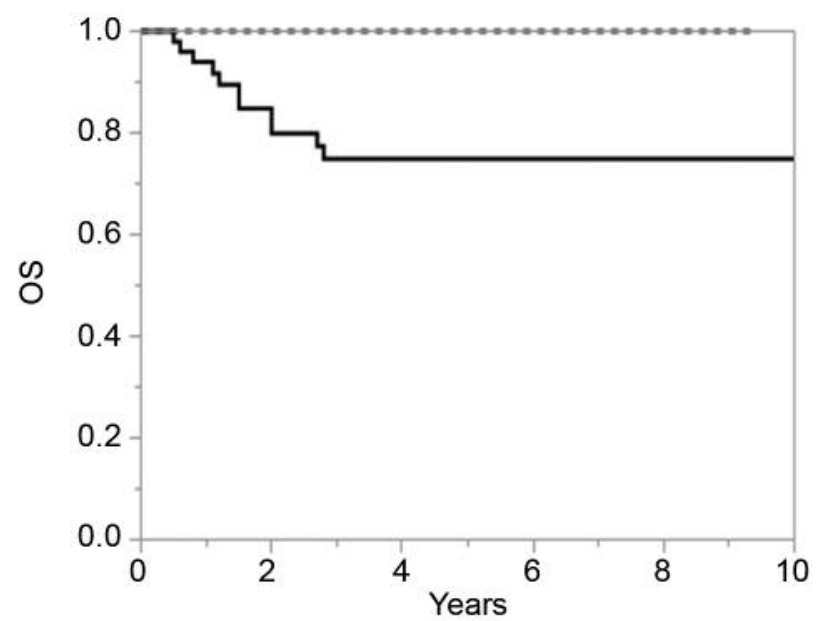

Figure 2. Overall survival in IPNB group and IPMN group. The 5-year survival rate of IPMN is $75 \%$, and of IPNB is 100\%. Dotted line: IPNB group; solid line: IPMN group.

All Authors approved the final version of the manuscript, and agreed to be accountable for all aspects of the work in ensuring that questions related to the accuracy or integrity of any part of the work are appropriately investigated and resolved.

\section{References}

1 Chen TC, Nakanuma Y, Zen Y, Chen MF, Jan YY, Yeh TS, Chiu CT, Kuo TT, Kamiya J, Oda K, Hamaguchi M, Ohno Y, Hsieh LL and Nimura Y: Intraductal papillary neoplasia of the liver associated with hepatolithiasis. Hepatology 34(4 Pt 1): 651-658, 2001. PMID: 11584359. DOI: 10.1053/jhep.2001.28199

2 Zen Y, Sasaki M, Fujii T, Chen TC, Chen MF, Yeh TS, Jan YY, Huang SF, Nimura Y and Nakanuma Y: Different expression patterns of mucin core proteins and cytokeratins during intrahepatic cholangiocarcinogenesis from biliary intraepithelial neoplasia and intraductal papillary neoplasm of the bile duct-an immunohistochemical study of 110 cases of hepatolithiasis. J Hepatol 44(2): 350-358, 2006. PMID: 16360234. DOI: 10.1016/ j.jhep.2005.09.025

3 Hisaka T, Horiuchi H, Uchida S, Ishikawa H, Kawahara R, Kawashima Y, Akashi M, Mikagi K, Ishida Y, Okabe Y, Nakayama M, Naito Y, Yano H, Taira T, Kawahara A, Kage M, Kinoshita $\mathrm{H}$ and Shirozu K: Potential usefulness of mucin immunohistochemical staining of preoperative pancreatic biopsy or juice cytology specimens in the determination of treatment strategies for intraductal papillary mucinous neoplasm. Oncol Rep 30(5): 2035-2041, 2013. PMID: 24008495. DOI: 10.3892/ or.2013.2720

4 Nakanuma Y, Curado MP and Franceschi S: Intrahepatic cholangiocarcinoma. In: WHO Classification of Tumours of the Digestive System; World Health Organization of Tumours. Bosman FT, Carneiro F, Hruban RH and Theise ND (eds.). 4th edition. Lyon: IARC, pp. 217-224, 2010.

5 Albores- Saavedra J, Adsay NV and Crawford JM: Carcinoma of the gallbladder and extrahepatic bile duct. In: WHO Classification of Tumours of the Digestive System; World Health Organization of Tumours. Bosman FT, Carneiro F, Hruban RH and Theise ND (eds.). 4th edition. Lyon: IARC, pp. 266-273, 2010.

6 Adsay NV, Fukushima N and Furukawa T: Intraductal Neoplasm of the pancreas. In: WHO Classification of Tumours of the Digestive System; World Health Organization of Tumours. Bosman FT, Carneiro F, Hruban RH and Theise ND (eds.). 4th edition. Lyon: IARC, pp. 304-313, 2010.

7 Fukumura Y, Nakanuma Y, Kakuda Y, Takase M and Yao T: Clinicopathological features of intraductal papillary neoplasms of the bile duct: a comparison with intraductal papillary mucinous neoplasm of the pancreas with reference to subtypes. Virchows Arch 471(1): 65-76, 2017. PMID: 28550497. DOI: 10.1007/s00428-017-2144-9

8 Nakanuma Y, Jang KT, Fukushima N, Furukawa T, Hong SM, Kim H, Lee KB, Zen Y, Jang JY and Kubota K: A statement by the Japan-Korea expert pathologists for future clinicopathological and molecular analyses toward consensus building of intraductal papillary neoplasm of the bile duct through several opinions at the present stage. J Hepatobiliary Pancreat Sci 25(3): 181-187, 2018. PMID: 29272078. DOI: 10.1002/jhbp.532

9 Tanaka M, Fernández-Del Castillo C, Kamisawa T, Jang JY, Levy P, Ohtsuka T, Salvia R, Shimizu Y, Tada M and Wolfgang CL: Revisions of international consensus Fukuoka guidelines for the management of IPMN of the pancreas. Pancreatology 17(5): 738753, 2017. PMID: 28735806. DOI: 10.1016/j.pan.2017.07.007

10 Nanashima A, Imamura N, Sumida Y, Hiyoshi M, Hamada T and Nagayasu T: clinicopathological aspects and diagnostic problems in patients with intraductal papillary neoplasm of the bile duct. Anticancer Res 38(4): 2343-2352, 2018. PMID: 29599359. DOI: 10.21873/anticanres.12481

11 Onoe S, Shimoyama Y, Ebata T, Yokoyama Y, Igami T, Sugawara G, Nakamura S and Nagino M: Prognostic delineation of papillary cholangiocarcinoma based on the invasive proportion: a singleinstitution study with 184 patients. Surgery 155(2): 280-291, 2014. PMID: 24287144. DOI: 10.1016/j.surg. 2013.08.011

12 Furukawa T, Hatori T, Fujita I, Yamamoto M, Kobayashi M, Ohike N, Morohoshi T, Egawa S, Unno M, Takao S, Osako M, Yonezawa S, Mino-Kenudson M, Lauwers GY, Yamaguchi H, Ban S and Shimizu M: Prognostic relevance of morphological types of intraductal papillary mucinous neoplasms of the pancreas. Gut 60(4): 509-516, 2011. PMID: 21193453. DOI: 10.1136/gut.2010.210567

13 Ikeno Y, Seo S, Yamamoto G, Nakamoto Y, Uemoto Y, Fuji H, Yoshino K, Yoh T, Taura K and Uemoto S: Usefulness of preoperative 18 F-FDG-PET in detecting invasive intraductal papillary neoplasm of the bile duct. Anticancer Res 38(6): 36773682, 2018. PMID: 29848727. DOI: 10.21873/anticanres.12645

14 Takanami K, Hiraide T, Tsuda M, Nakamura Y, Kaneta T, Takase K, Fukuda $\mathrm{H}$ and Takahashi S: Additional value of FDG PET/CT to contrast-enhanced CT in the differentiation between benign and malignant intraductal papillary mucinous neoplasms of the pancreas with mural nodules. Ann Nucl Med 25(7): 501-510, 2011. PMID: 21537945. DOI: 10.1007/s12149-011-0494-y

Received May 23, 2019

Revised June 26, 2019

Accepted June 27, 2019 